\title{
Relevance Vector Machine Based Analyses of MRR and SR of Electrodischarge Machining Designed by Response Surface Methodology
}

\author{
Kanhu Charan Nayak, ${ }^{1}$ Rajesh Kumar Tripathy, ${ }^{1}$ and Sudha Rani Panda ${ }^{2}$ \\ ${ }^{1}$ National Institute of Technology, Rourkela 769008, India \\ ${ }^{2}$ Biju Patnaik University of Technology, Rourkela 769004, India \\ Correspondence should be addressed to Kanhu Charan Nayak; nayakkanhu83@gmail.com
}

Received 26 June 2013; Accepted 19 November 2013

Academic Editors: G. Dessein, J.-Y. Hascoet, A. Lockamy, and H. Yu

Copyright (C) 2013 Kanhu Charan Nayak et al. This is an open access article distributed under the Creative Commons Attribution License, which permits unrestricted use, distribution, and reproduction in any medium, provided the original work is properly cited.

\begin{abstract}
Relevance vector machine is found to be one of the best predictive models in the area of pattern recognition and machine learning. The important performance parameters such as the material removal rate (MRR) and surface roughness (SR) are influenced by various machining parameters, namely, discharge current $\left(I_{p}\right)$, pulse on time $\left(T_{\text {on }}\right)$, and duty cycle (tau) in the electrodischarge machining process (EDM). In this communication, the MRR and SR of EN19 tool steel have been predicted using RVM model and the analysis of variance (ANOVA) results were performed by implementing response surface methodology (RSM). The number of input parameters used for the RVM model is discharge current $\left(I_{p}\right)$, pulse on time $\left(T_{\text {on }}\right)$, and duty cycle (tau). At the output, the corresponding model predicts both MRR and SR. The performance of the model is determined by regression test error which can be obtained by comparing both predicted MRR and SR from model and experimental data is designed using central composite design (CCD) based RSM. Our result shows that the regression error is minimized by using cubic kernel function based RVM model and the discharge current is found to be one of the most significant machining parameters for MRR and SR from ANOVA.
\end{abstract}

\section{Introduction}

Electrodischarge machining (EDM) has tremendous potentials on account of its versatile application in industry. The applications are like high precision machining of all types of electrical conductors and hard material such as metal and metal alloys like tool steel and steels used for die making in metal forming processes and manufacturing of molds, automotive, aerospace, and surgical components. It has enormous advantages without any physical contact between tool and work piece. This nonconventional machining process uses the thermoelectric energy for machining and removes the material by thermal erosion process. The EDM process involves finite discrete periodic electric sparks created by the electric pulse generator at short intervals between the tool electrode (anode) and work electrode (cathode) separated by a thin film of dielectric liquid that causes the material removal in motion and vaporize form, and these tiny molten vaporize particles are flushed away from the gap by continuous flushing of dielectric liquid. This machining process provides productive with increasing strength of the work material and maintains desire shape, accuracy, and surface integrity requirements [1]. Material removal rate (MRR) and surface roughness (SR) are the most important responses which are influenced by EDM parameters such as voltage gap, discharge current, pulse on time, pulse off time, electrode gap, and duty factor. So it is important to determine the relationship between various responses (MRR and SR) with machining criteria (machining process parameters) and explore the effects of these process parameters on the responses. For this, a collection of mathematical and statistical techniques called response surface methodology (RSM) is used. RSM is an empirical modelling approach useful for modelling and analysis of problems in which responses are influenced as several variables and objectives to optimize these responses [2]. 
A number of studies have been investigated for material removal rate and surface roughness in EDM process and effect of machining parameters on machining performance. The modelling of material removal rate through RSM, Rahman et al. [3] conclude that the MRR is influenced considerably by peak current and pulse on time. Khan et al. [4] showed a mathematical model for investigation of process parameters (peak current, pulse on time, and pulse off time) on response parameter surface roughness with Ti-6Al-4V as work piece material and copper as an electrode in electrodischarge machining. They conducted a complete analysis of variance (ANOVA) to know the optimal process parameters and prediction of roughness using RSM. Central composite design based RSM is used is relatively efficient and used for to design the experiment and the investigation of machining parameters on the performance of EDM process. It helps the modelling of process parameters [5]. RSM is applied for modelling and prediction of roughness in the electrodischarge machining process for EN8 steel considering discharge current and other machining parameters. The most influencing factor in this study was found to be discharging current [6]. Another notable study by Prabhu and Vinayagam [7] was on the modelling and characterization of EDM process parameters of AISI D2 toll steel material with multiwall carbon nanotubes and developed the prediction of SR as response parameter and pulse current, pulse duration, and pulse voltage as model variables with copper electrode using Box-Behnken in RSM. Li and Lee [8] studied the different optimal machining parameters (voltage, discharge current, pulse on time, and flushing pressure) setting and electrode polarity (copper tungsten electrode) on response parameter MRR and SR by implementing RSM. Tsai and Wang [9] have carried out the works to study the neural network based as RBFN and ANFIS for the prediction of surface finish in EDM aluminum and iron as work piece and copper as an electrode.

Those above mentioned investigations were restricted to studying the effect of various discharge machining parameters on the MRR and SR in EDM with EN19 tool steel and other machining parameters called duty cycle. Hence the present study attempts to investigate the machining performance using EN19 as work piece material. EN19 has tremendous application towards the components of mediums and large cross section, requiring high tensile strength and toughness for automatic engineering and gear and engine construction such as crane shafts steering knocking connecting rods.

Although the response surface methodology and other prediction tools are a powerful approach for the investigation of material removal rate and the surface roughness during the EDM process, it is time-consuming. During recent decades, the RVM was been a popular machine learning tool for solving complex problems. In this model a general Bayesian framework is used for obtaining the sparse solutions to regression (function estimation) and classification tasks by utilizing linear models in the parameters [10]. The Bayesian approach has the advantage that it can be seamlessly incorporated into the RVM framework and thus it inherits the subsequent advancement made towards the faster computations from a practical standpoint [11]. Candela and Hansen [12] summarized the Bayesian framework used to train RVM,

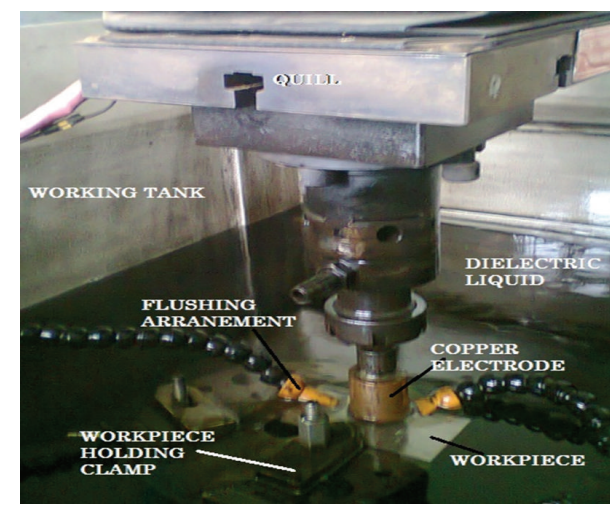

FIGURE 1: Experimental setup (electrode, work piece, and flushing arrangement).

highlighted the importance of adapting the basic functions, and presented the improvement to the RVM. Caesarendra et al. [13] used RVM to train the kurtosis bearing data and target vectors of failure probability estimated by LR and employed to predict the failure probability of the individual unit of bearing sample. Valls et al. [14] introduced a general class of RVMbased system identification algorithms and yield confidence intervals for the predictions.

It was concluded from the above study that limited work has been done on EDM of EN19 (alloy steel) and use of RVM model to predict EDM responses namely MRR and SR. Therefore it is needed to work out the present research for EN19 as work piece material and RVM model as the machine learning algorithim. The present study was initiated to develop a multiinput-multioutput RVM model to predict the values of MRR and SR resulting from an EDM process. The three process parameters, namely, discharge current, pulse on time, and duty cycles, were varied to investigate their effect on response parameters such as material removal rate and surface roughness. The process optimizations of these parameters were done by response surface methodology. In this paper we use the application of large scale multikernel RVM for the prediction of MRR and SR for EDM process.

\section{Experimental Details}

2.1. Experimental Setup. The experiments are carried out utilizing LEADER-1 ZNC electrical discharge machine. The EDM has provision of movement in three axes, $X$ - and $Y$ axis movement of working table and $Z$-axis movement of the quill. In this effort EN19 was selected as the work piece material and cylindrical copper electrode were employed for machining the work piece material. Three process parameters, discharge current, pulse on time, and duty factor at different levels, are taken to carry out the machining process keeping other input parameters (voltage $=40$ volt, $\mathrm{SEN}=6$, ASEN $=3$ working cycle $=0.8 \mathrm{sec}$, and quill-up time $0.3 \mathrm{Sec}$ ) as consultant for each machining operation and to evaluate the output responses, MRR and SR. Impulse flushing is used with a flushing pressure of $0.2 \mathrm{Kg} / \mathrm{cm}^{2}$. The experimental setup is shown in Figure 1. The chemical composition and 
mechanical properties of the work piece is given in Tables 1 and 2 , respectively.

The machining was carried out for a fixed depth of machining of $0.2 \mathrm{~mm}$ and for each depth of machining the time is measured by using a digital stopwatch. The experimental schedules are shown in Table 4 which is designed by RSM. The weight of work piece before and after machining was measured by digital balance. The initial and final weight of the work piece after end of each machining and the density of work piece are used for the calculation of MRR by using (1). Surface roughness was measured after each machining by using a portable stylus type profile meter, Talysurf with sample length $0.8 \mathrm{~mm}$, filter $2 \mathrm{CR}$, evaluation length $4 \mathrm{~mm}$, and traverse speed $1 \mathrm{~mm} / \mathrm{Sec}$. All the response parameters, MRR and SR, are tabulated in Table 4,

$$
\operatorname{MRR}=\frac{\left(W_{0}-W_{1}\right) \times 10^{-3} \times 10^{9}}{\rho_{w} \times \text { time }} \text { in } \mathrm{mm}^{3} / \mathrm{min}
$$

where $W_{0}=$ initial weight in $\mathrm{Kg}, W_{1}$ = weight after one machining in $\mathrm{Kg}$, and $\rho_{w}=$ density of material in $\mathrm{Kg} / \mathrm{m}^{3}$.

2.2. Design of Experiment. The design of experiments for exploring the effect of various predominant EDM process parameters (e.g., Pulse on time, discharge current, and duty cycle) on the machining characteristics (e.g., the material removal rate and surface roughness) was modelled. The main objective of experimental design is to study the relations between the response as a dependent variable and the various process parameter levels. It provides an opportunity to study the individual effects of each factor.

In the present work experiments were designed on the basis of experimental design technique using RSM that is a mathematical and statistical techniques which are useful for the modelling and analysis of an experiment in which a response of interest is influenced by several variables and the objective is to optimize the response. The coded levels for all process parameters used are displayed in Table 3 . To obtain an optimal response, a central composite design (CCD) type of design is implemented in RSM which is presented in Table 4. A CCD is used since it gives a comparatively accurate prediction of all response variable averages related to quantities measured during experimentation and relatively efficient with respect to the number of runs required [15].

\section{RVM Modelling}

Relevance vector machine (RVM) is a machine learning technique based on a Bayesian formulation of a linear model with Proper selection of prior that result in a sparse representation RVM is a special type of a sparse linear model, in which the basis functions are formed by using kernel functions centered at the different training points $[10,16]$. For this model the $m_{i}$ 's are the input parameters, $w_{i}$ are the weight vectors from hidden feature space to output, and $N$ is the dimension of feature space. The required block diagram of RVM model is shown in Figure 2.

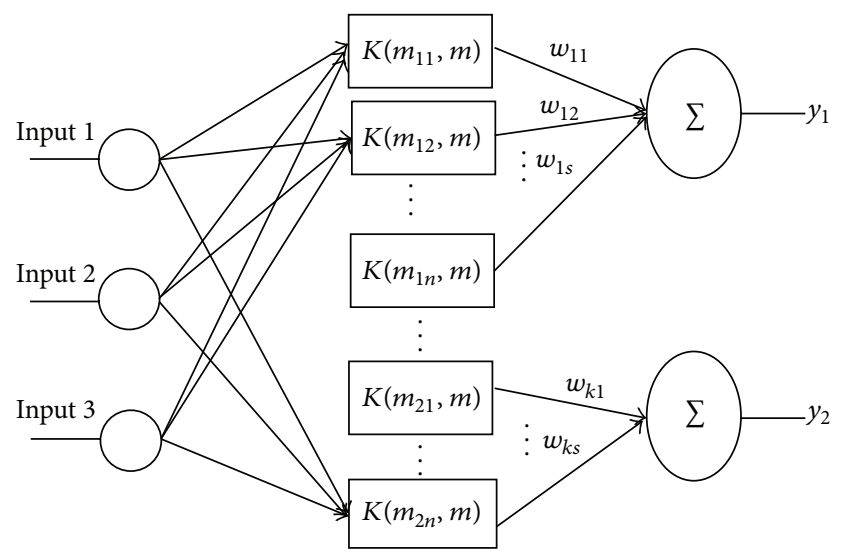

FIgURE 2: Shows the block diagram of RVM. $K\left(m_{11}, m\right), \ldots$, $K\left(m_{1 n}, m\right)$ and $K\left(m_{21}, m\right), \ldots, K\left(m_{2 n}, m\right)$ : Kernel functions for $m_{n}$ input features. $w$ : weight vector. $y_{1}$ and $y_{2}$ : output responses (for MRR and SR).

TABLE 1: Chemical composition of work piece (EN19).

\begin{tabular}{lc}
\hline Elements & Percentage of weight \\
\hline $\mathrm{C}$ & $0.38-0.43$ \\
$\mathrm{Mn}$ & $0.75-1.00$ \\
$\mathrm{P}$ & 0.035 \\
$\mathrm{~S}$ & 0.04 \\
$\mathrm{Si}$ & $0.15-0.3$ \\
$\mathrm{Cr}$ & $0.8-1.10$ \\
$\mathrm{Mo}$ & $0.15-0.25$ \\
\hline
\end{tabular}

TABLE 2: Mechanical properties of work piece (EN19).

\begin{tabular}{lc}
\hline Density $\left(\mathrm{Kg} / \mathrm{m}^{3}\right)$ & $7.7 \times 10^{3}$ \\
Poisson's ratio & $0.27-0.3$ \\
Elastic modulus (GPA) & $190-210$ \\
Hardness (HB) & 197 \\
\hline
\end{tabular}

TABLE 3: EDM process parameters and their levels assigned for experiment.

\begin{tabular}{lcccccc}
\hline \multirow{2}{*}{ Process parameters } & \multirow{2}{*}{ Unit } & \multicolumn{5}{c}{ Levels } \\
& & -2 & -1 & 0 & 1 & 2 \\
\hline Discharge current $\left(I_{p}\right)$ & Ampere & 1 & 2 & 4 & 7 & 8 \\
Pulse on time $\left(T_{\text {on }}\right)$ & Ms & 50 & 200 & 500 & 750 & 1000 \\
Duty cycle $(\mathrm{tau})$ & $\%$ & 45 & 55 & 65 & 80 & 85 \\
\hline
\end{tabular}

The output function $y(m)$ is defined as follows:

$$
y(m)=\sum_{i=1}^{N} w_{i} \phi\left(m-m_{i}\right)
$$

Basically the RVM model is identical to that of support vector machines (SVM) but here the kernel function does not satisfy Mercer's condition [10]. The kernel function " $\phi$ " is a continuous symmetric positive integral operator.

Let us assume the sparse Bayesian regression model and associated inference procedures to predict both MRR and 
TABLE 4: CCD for process parameters using RSM and experimental results.

\begin{tabular}{|c|c|c|c|c|c|}
\hline Sl. no. & $I_{p}$ & $T_{\text {on }}$ & tau & $\operatorname{MRR}\left(\mathrm{mm}^{3} / \mathrm{min}\right)$ & $\mathrm{SR}(\mu \mathrm{m})$ \\
\hline 1 & 2.00 & 750.00 & 55.00 & 0.31107 & 3.72 \\
\hline 2 & 7.00 & 750.00 & 80.00 & 4.62736 & 7.8 \\
\hline 3 & 2.00 & 200.00 & 80.00 & 1.77819 & 4.17 \\
\hline 4 & 4.00 & 500.00 & 65.00 & 2.68492 & 5.13 \\
\hline 5 & 4.00 & 500.00 & 65.00 & 2.59718 & 4.79 \\
\hline 6 & 7.00 & 200.00 & 55.00 & 3.97481 & 11.27 \\
\hline 7 & 7.00 & 750.00 & 55.00 & 4.57803 & 9.12 \\
\hline 8 & 4.00 & 500.00 & 65.00 & 2.69626 & 5.2 \\
\hline 9 & 4.00 & 500.00 & 65.00 & 2.65843 & 5.21 \\
\hline 10 & 2.00 & 750.00 & 80.00 & 0.39685 & 3.8 \\
\hline 11 & 7.00 & 200.00 & 80.00 & 4.06222 & 10.33 \\
\hline 12 & 2.00 & 200.00 & 55.00 & 1.8393 & 3.53 \\
\hline 13 & 4.00 & 500.00 & 65.00 & 2.78798 & 4.7 \\
\hline 14 & 8.00 & 500.00 & 65.00 & 5.404 & 11.43 \\
\hline 15 & 4.00 & 50.00 & 65.00 & 2.43872 & 5.83 \\
\hline 16 & 4.00 & 1000.00 & 65.00 & 0.76172 & 4.13 \\
\hline 17 & 4.00 & 500.00 & 65.00 & 2.79229 & 4.87 \\
\hline 18 & 1.00 & 500.00 & 65.00 & 0.6589 & 3.47 \\
\hline 19 & 4.00 & 500.00 & 85.00 & 2.69359 & 4.73 \\
\hline
\end{tabular}

SR for EDM process. The experimental data set of inputoutput pairs obtained from RSM analysis are given in form of $\left\{m_{n}, t_{n}\right\}_{n=1}^{N}$, where " $m_{n}$ " are the input features and " $t_{n}$ " are the output features. By considering only the scalar valued output we follow the standard probabilistic formulation and add additive noise with output samples for better data overfitting, which is described as follows:

$$
t_{n}=y\left(m_{n} ; w\right)+\varepsilon_{n},
$$

where $\varepsilon_{n}$ independent samples from some noise are processed and assumed to be zero-mean Gaussian noise with variance $\sigma^{2}$. Thus the probability function defines the noise as $p\left(t_{n} \mid\right.$ $m)=N\left(t_{n} \mid y\left(m_{n}\right), \sigma^{2}\right)$. This probability distribution specifies a Gaussian distribution over the output $t_{n}$ with mean value as $y\left(m_{n}\right)$ and variance $\sigma^{2}[17,18]$.

Due to the assumption of independence of the likelihood function, the complete data set can be written as follows:

$$
p\left(t \mid w, \sigma^{2}\right)=\frac{1}{\left(2 \pi \sigma^{2}\right)^{-(N / 2)}} \exp \left\{-\frac{1}{2 \sigma^{2}}\|t-\phi w\|^{2}\right\},
$$

where $t=\left(t_{1}, \ldots, t_{N}\right)^{T}$ are the output vectors, $w=\left(w_{0}, \ldots\right.$, $\left.w_{N}\right)^{T}$ are weight vectors, and $\phi$ is the $N \times(N+1)$ design matrix. The $\phi$ is

$$
\phi\left(m_{n}\right)=\left[1, K\left(m_{n}, m_{1}\right), K\left(m_{n}, m_{2}\right), \ldots, K\left(m_{n}, m_{N}\right)\right]^{T} .
$$

Here the $K$ specifies the kernel function which maps the input features to high dimensional feature space just alike as SVM.
Here we used different kernel fuctions like Gaussian kernel, laplace kernel, and spline kernel. The different parameters in the model obtained from training examples are $w$ and $\sigma^{2}$. We expect a normalized value of both $w$ and $\sigma^{2}$ for better prediction of testing data.

To modify this approach we should follow the Bayesian prior probability distribution. At first we encode a preference for smoother functions by making the popular choice of a zero-mean Gaussian prior distribution over $w$. The distribution is given as follows:

$$
p\left(\frac{w}{\alpha}\right)=\prod_{i=0}^{N} N\left(w_{i} \mid 0, \alpha_{i}^{-1}\right)
$$

where $\alpha$ is a vector of $N+1$ hyper parameters. The hyper parameters are associated with every weight between hidden feature and output.

The Bayesian inference obtained from Bay's rule, which is given by the following

$$
p\left(w, \alpha, \sigma^{2} \mid t\right)=\frac{p\left(t \mid w, \alpha, \sigma^{2}\right) p\left(w, \alpha, \sigma^{2}\right)}{p(t)} .
$$

The new given test point $m_{*}$ can be predicted with respect to target $t_{*}$ in terms of predictive distribution as follows:

$$
p\left(t_{*} \mid t\right)=\int p\left(t_{*} \mid w, \alpha, \sigma^{2}\right) p\left(w, \alpha, \sigma^{2} \mid t\right) d w d \alpha d \sigma^{2} .
$$


The second term in the integral called as posterior distribution over weight which is given by

$$
\begin{aligned}
p\left(w \mid t, \alpha, \sigma^{2}\right) & =\frac{p\left(t \mid w, \sigma^{2}\right) p(w \mid \alpha)}{p\left(t \mid \alpha, \sigma^{2}\right)} \\
& =(2 \pi)^{-(N+1) / 2} \\
& \times\left|\sum\right|^{-1 / 2} \exp \left\{-\frac{1}{2}(w-\mu)^{T} \sum^{-1}(w-\mu)\right\} .
\end{aligned}
$$

The posterior covariance term is given by the following:

$$
\begin{gathered}
\sum=\left(\sigma^{-2} \Phi^{T} \Phi+A\right)^{-1}, \\
\mu=\sigma^{-2} \sum \Phi^{T} t
\end{gathered}
$$

with $A=\operatorname{diag}\left(\alpha_{0}, \alpha_{1}, \alpha_{2}, \ldots, \alpha_{N}\right)$.

Relevance vector machine method is a learning procedure to search for the best hyper parameters posterior mode that is, the maximization of $p\left(\alpha, \sigma^{2} \mid t\right)$ proportional to $p\left(t \mid \alpha, \sigma^{2}\right) p(\alpha) p\left(\sigma^{2}\right)$ with respect to $\alpha$ and $\beta$; in case of the uniform hyper priors we need to maximize the term $p\left(t \mid \alpha, \sigma^{2}\right)$ [19]. The maximization term can be computed as follows:

$$
\begin{aligned}
p\left(t \mid \alpha, \sigma^{2}\right)= & \int p\left(t \mid w, \sigma^{2}\right) p(w \mid \alpha) d w \\
= & (2 \pi)^{-N / 2}\left|\sigma^{2} I+\phi A^{-1} \phi^{T}\right|^{-1 / 2} \\
& \times \exp \left\{-\frac{1}{2} t^{T}\left(\sigma^{2} I+\phi A^{-1} \phi^{T}\right)^{-1} t\right\} .
\end{aligned}
$$

Values of $\alpha$ and $\sigma^{2}$ which maximize (11) cannot be obtained in closed form, and here we summarize formulae for their iterative estimation. The detail information regarding the estimation of hyperparameters can be obtained using expectation maximization algorithm based approach.

For $\alpha$, differentiating (11) and equating to zero we get the value of $\alpha_{i}^{\text {new }}$ as follows:

$$
\alpha_{i}^{\text {new }}=\frac{\gamma_{i}}{\mu_{i}^{2}}
$$

where $\mu_{i}$ is the $i$ th posterior mean weight. Thus we can define the quantities $\gamma_{i}$ as follows:

$$
\gamma_{i} \equiv 1-\alpha_{i} \sum_{i i}
$$

where the $\sum_{i i}$ is the $i$ th diagonal element of the posterior weight covariance from (8) computed with the current $\alpha$ and $\sigma^{2}$ values. For the noise variance $\sigma^{2}$, differentiation leads to the reestimate as follows:

$$
\left(\sigma^{2}\right)^{\text {new }}=\frac{t-\Phi \mu^{2}}{N-\sum_{i} \gamma_{i}},
$$

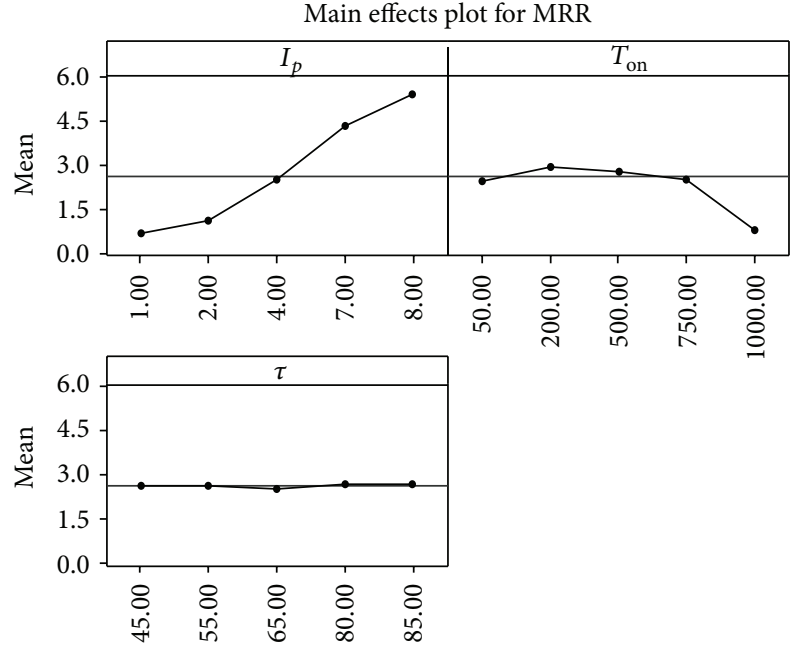

FIGURE 3: Main effects of machining parameters on MRR.

where $N$ in the denominator refers to the number of data examples.

During the convergence of the hyper parameter estimation procedure, we have to make predictions based on the posterior distribution over the weights, in which the conditions of the maximizing values are $\alpha_{\mathrm{MP}}$ and $\sigma_{\mathrm{MP}}^{2}$ [19]. We can then compute the predictive distribution, for a new data $m_{*}$ by using $(8)$ :

$$
\begin{aligned}
& p\left(t_{*} \mid t, \alpha_{\mathrm{MP}}, \sigma_{\mathrm{MP}}^{2}\right) \\
& \quad=\int p\left(t_{*} \mid w, \sigma_{\mathrm{MP}}^{2}\right) p\left(w \mid t, \alpha_{\mathrm{MP}}, \sigma_{\mathrm{MP}}^{2}\right) d w .
\end{aligned}
$$

As both terms in the integral are Gaussian, so we can compute the probability as follows:

$$
p\left(t_{*} \mid t, \alpha_{\mathrm{MP}}, \sigma_{\mathrm{MP}}^{2}\right)=N\left(t_{*} \mid y_{*}, \sigma_{*}^{2}\right)
$$

with

$$
\begin{gathered}
y_{*}=\mu^{T} \phi\left(m_{*}\right) \\
\sigma_{*}^{2}=\sigma_{\mathrm{MP}}^{2}+\phi\left(m_{*}\right)^{T} \sum \phi\left(m_{*}\right) .
\end{gathered}
$$

So the prediction of the output values (MRR and SR) denoted by $y\left(m_{*} ; \mu\right)$ in $(2)$ can be computed by taking the normalized values of $\sigma_{*}^{2}$ and $\phi\left(m_{*}\right)$.

\section{Result and Discussion}

ANOVA and main effect plots for MRR and SR are done by response surface methodology using MINTAB 16. And with the help of 40 sets of experimental input-output patterns, the proposed RVM modelling are carried out. The software programs for RVM model are implemented using MATLAB version 10.1. 
TABLE 5: Analysis of variance (ANOVA) for MRR and SR.

\begin{tabular}{|c|c|c|c|c|c|c|}
\hline \multirow{2}{*}{ Source } & \multicolumn{3}{|c|}{$\operatorname{MRR}\left(\mathrm{mm}^{3} / \mathrm{min}\right)$} & \multicolumn{3}{|c|}{$\mathrm{SR}(\mu \mathrm{m})$} \\
\hline & $F$ & $P$ & $\%$ of contribution & $F$ & $P$ & $\%$ of contribution \\
\hline$I_{p}$ & 10377.67 & 0.000 & 84.34 & 2145.29 & 0.000 & 85.84 \\
\hline$T_{\text {on }}$ & 377.47 & 0.000 & 4.13 & 120.39 & 0.000 & 3.84 \\
\hline tau & \multicolumn{3}{|c|}{ Insignificant } & 14.87 & 0.002 & 0.224 \\
\hline$I_{p} * I_{p}$ & & & & 199.47 & 0.000 & 6.77 \\
\hline$T_{\text {on }} * T_{\text {on }}$ & 653.71 & 0.000 & 5.8 & \multicolumn{3}{|c|}{ Insignificant } \\
\hline$I_{p} * T_{\mathrm{on}}$ & 662.65 & 0.000 & 5.58 & 60.75 & 0.000 & 2.04 \\
\hline \multirow[t]{2}{*}{$I_{p} *$ tau } & \multicolumn{3}{|c|}{ Insignificant } & 24.67 & $I_{p} *$ tau & 0.84 \\
\hline & \multicolumn{3}{|c|}{$S=0.05698, R^{2}=99.87, R^{2}($ adj $)=99.84$} & \multicolumn{3}{|c|}{$S=0.20722, R^{2}=99.56, R^{2}(\operatorname{adj})=99.36$} \\
\hline
\end{tabular}

$F:$ it is a test to determine whether the interaction and main effects are significant.

$P$ : probability of obtaining a test statistic that at least as extreme as the actual calculated value, if the null hypothesis is true.

$S:$ standard deviation error.

$R^{2}$ : the amount of variation seen in MRR and SR and is explained by input factor.

$R^{2}$ (adj): modified $R^{2}$ that has been adjusted for a number of terms in the model.

TABLE 6: Regression test error for different kernel functions.

\begin{tabular}{lcc}
\hline Kernel functions & $\begin{array}{c}\text { Noise factor }=0.1, \text { number of iterations }=100 \\
\text { Regression test error for MRR }\end{array}$ & Regression test error for SR \\
\hline Gaussian kernel & 0.083636 & 0.079863 \\
Laplace kernel & 0.077997 & 0.074544 \\
Linear spline kernel & 0.088783 & 0.078388 \\
Cubic kernel & 0.070611 & 0.066244 \\
Distance kernel & 0.129122 & 0.103465 \\
Thin-plate spline & 0.086694 & 0.075625 \\
Neighborhood indication & 0.257006 & 0.220205 \\
\hline
\end{tabular}

4.1. ANOVA and Main Effect Plots. The influences of various machining parameters on MRR are shown in Figure 3. The discharge current has a highly significant effect on MRR and it has directly proportional effect. MRR is increasing with pulse on time up to approximately $200 \mu$ s and then decreases with increasing value of pulse on time. That is, MRR increases with pulse on time then afterwards it decreases; however according to the abridge ANOVA, Table 5 effects are less than the discharge current on MRR. The surface roughness is not much more important in case of roughing operation and required high MRR. For this point of view the pulse on time (about $200 \mu \mathrm{s}$ ) gives the advantages in saving the machining time in EDM ultimately which reduce the cost of product. So, it is not required to take the high value of $T_{\text {on }}$ which is more than this optimum value (about $200 \mu \mathrm{s}$ ). But MRR decreases with further increasing of $T_{\text {on }}$. It may be due to decrease of flushing time with increasing $T_{\text {on }}$, which causes the debris recast on machining surface. Hence MRR decreases. So it is required to choose an optimum machining parameter. The percentages of contributions are 84.34 and $4.13 \%$ for discharge current and pulse on time, respectively, as shown in Table 5.

The effects of various input parameters on SR are shown in Figure 4. The graph shows that the Ip is proportional to SR and it is highly significant and has more percentage of contribution towards the SR. Surface roughness also is affected by pulse on time duty factor with less contribution than discharge current. Pulse on time influences the SR in the same manner as it affects the MRR. As discussed earlier, the larger value of pulse on time decreases the MRR, but it may improve the surface quality by filling the crater with molten metal called recast layer. However this recast layer affects the mechanical and metallurgical properties of machined component, which is treated as one of the disadvantage [20]. This is explained by the fact that to minimize the SR, Ton may be taken as less than $200 \mu$ s and also for low value of $I_{p}(1 \mathrm{~A}$ to $2 \mathrm{~A}$ ). The percentages of contribution of $I_{p}, T_{\text {on }}$, and tau are $85.84,3.84$, and $0.224 \%$ as shown in Table 5 .

4.2. Prediction of MRR and SR by RVM. The proposed modelling is carried out by relevance vector machine (RVM) with the help of 40 sets of experimental input-output patterns obtained from RSM (response surface methodologies) in EDM process. As lesser amount of data available from the experimental design we again sampled the data and further used those data for both training and testing of RVM model. The different machining parameters such as discharge current $\left(I_{P}\right)$, pulse on time $\left(T_{\text {on }}\right)$ and duty cycle (tau) are the input to RVM model. At the output, the respective RVM model predicts parameters like material removal rate (MRR) and 
TABLE 7: Regression test error for different noise factors.

\begin{tabular}{lcc}
\hline & $\begin{array}{c}\text { For cubic kernel function, number of iterations }=100 \\
\text { Regression test error for MRR }\end{array}$ & Regression test error for SR \\
\hline 0.05 & 0.037754 & 0.035251 \\
0.01 & 0.012026 & 0.018637 \\
\hline
\end{tabular}

TABLE 8: Regression test error for number of iterations.

\begin{tabular}{lcr}
\hline No. of iterations & \multicolumn{2}{c}{$\begin{array}{c}\text { For cubic kernel function, noise factor }=0.01 \\
\text { Regression test error for MRR }\end{array}$} \\
\hline 100 & 0.012026 & Regression test error for SR \\
200 & 0.010694 & 0.018637 \\
250 & 0.01293 & 0.015266 \\
\hline
\end{tabular}
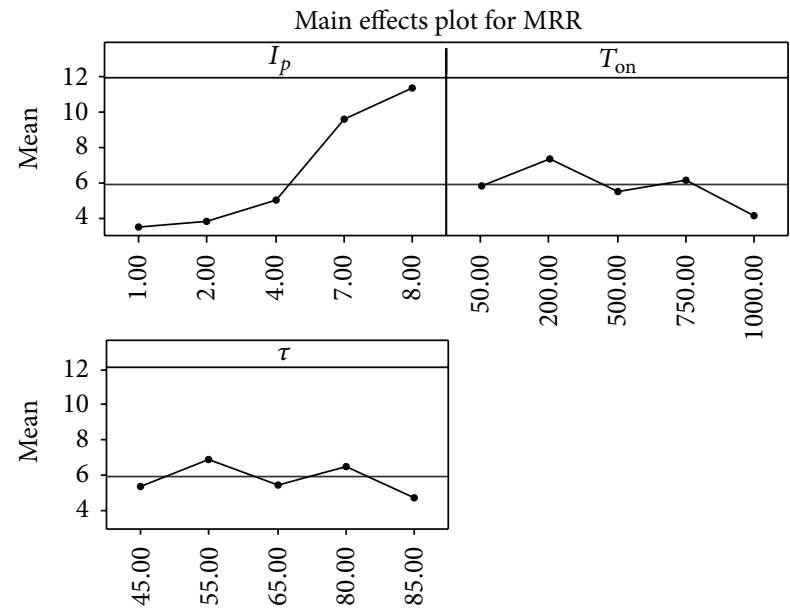

FIGURE 4: Main effects of machining parameters on SR.

surface roughness (SR). The performance of RVM modelling is obtained from regression test error. The regression test error for RVM prediction using different kernel function with the number of iterations $\left(N_{t}\right)$ and noise factor is given in Tables 6, 7, and 8. The lower regression error corresponds to the better prediction of MRR and SR for the respective RVM model.

From Table 6 it is quite clear that with number of iterations $N=100$ and noise factor as 0.1 , the cubic kernel function is the optimized one and gives a lower value of regression test error. Further from Table 7, by decreasing the noise factor from 0.1 to 0.01 we get better prediction of both MRR and SR with a low value of regression test error. From Table 8, it is followed that further by increasing the number of iterations to 200 at a constant noise factor the respective value of regression test error is minimized. From these above results it is observed that, the better prediction performance of model depends upon the regression error. The different kernel functions and the nature of experiment performed play an important role in designing the best regression model. From our previous paper that is for the prediction of MRR and SR for electrochemical machining, we obtain the result as follows. The minimum regression test

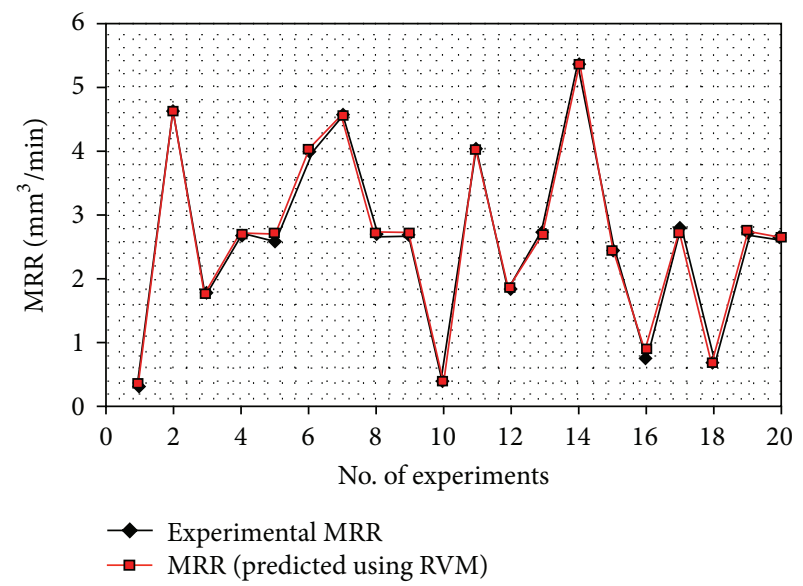

FIGURE 5: The comparison between predicted MRR and experimental MRR.

error for both MRR and SR is minimized by the help of laplace kernel function with 0.001 noise factor [18]. It may give the idea to model RVM taking any experimental data for prediction of responses with the above said kernel function and then other kernel, which minimize the computational time. Prediction of SR and MRR by RVM gives low error as compared to the least square support vector machine and artificial neural network based predictive model for ECM process [21]. Rather conducting large number of experiments, we can use proposed model for predicting the responses. This can save the time and cost required for performing the number of experiments. In Table 9 and Figures 5 and 6 we compare the results of both experimental data and predicted data of both MRR and SR.

\section{Conclusion}

The experimental design of EDM was successfully implemented using CCD based on RSM. From the RSM analysis, the most significant parameter towards the response was found to be the discharging current. The proper selection of pulse time can save the machining time as well as cost of 
TABLE 9: Experimental and predicted values of MRR and SR.

\begin{tabular}{|c|c|c|c|c|c|c|}
\hline$I_{p}$ & $T_{\text {on }}$ & tau & $\begin{array}{c}\mathrm{MRR} \\
\left(\mathrm{mm}^{3} / \mathrm{min}\right) \\
\end{array}$ & $\mathrm{SR}(\mu \mathrm{m})$ & $\begin{array}{l}\text { MRR (predicted using } \\
\text { RVM) in } \mathrm{mm}^{3} / \mathrm{min}\end{array}$ & $\begin{array}{c}\text { SR (predicted } \\
\text { using RVM) in } \mu \mathrm{m}\end{array}$ \\
\hline 2.0 & 750.00 & 55.00 & 0.311073 & 3.72 & 0.33439221 & 3.710796635 \\
\hline 7.00 & 750.00 & 80.00 & 4.627355 & 7.8 & 4.602598191 & 7.90183655 \\
\hline 2.00 & 200.00 & 80.00 & 1.77819 & 4.17 & 1.775926522 & 4.169651963 \\
\hline 4.00 & 500.00 & 65.00 & 2.684917 & 5.13 & 2.700207547 & 4.943501339 \\
\hline 4.00 & 500.00 & 65.00 & 2.59718 & 4.79 & 2.700207547 & 4.943501339 \\
\hline 7.00 & 200.00 & 55.00 & 3.974812 & 11.27 & 4.014896139 & 11.20501356 \\
\hline 7.00 & 750.00 & 55.00 & 4.578032 & 9.12 & 4.540240762 & 8.886500507 \\
\hline 4.00 & 500.00 & 65.00 & 2.69626 & 5.2 & 2.700207547 & 4.943501339 \\
\hline 4.00 & 500.00 & 65.00 & 2.658429 & 5.21 & 2.700207547 & 4.943501339 \\
\hline 2.00 & 750.00 & 80.00 & 0.39685 & 3.8 & 0.379393774 & 3.585126803 \\
\hline 7.00 & 200.00 & 80.00 & 4.062215 & 10.33 & 4.005057172 & 10.32266034 \\
\hline 2.00 & 200.00 & 55.00 & 1.839296 & 3.53 & 1.868379133 & 3.56482374 \\
\hline 4.00 & 500.00 & 65.00 & 2.78798 & 4.7 & 2.700207547 & 4.943501339 \\
\hline 8.00 & 500.00 & 65.00 & 5.404 & 11.43 & 5.343913023 & 11.43558399 \\
\hline 4.00 & 50.00 & 65.00 & 2.43872 & 5.83 & 2.426300736 & 5.825788079 \\
\hline 4.00 & 1000.00 & 65.00 & 0.761718 & 4.13 & 0.881463669 & 4.105567142 \\
\hline 4.00 & 500.00 & 65.00 & 2.792288 & 4.87 & 2.700207547 & 4.943501339 \\
\hline 1.00 & 500.00 & 65.00 & 0.6589 & 3.47 & 0.672458618 & 3.782897979 \\
\hline 4.00 & 500.00 & 85.00 & 2.69359 & 4.73 & 2.745193125 & 4.986478772 \\
\hline 4.00 & 500.00 & 45.00 & 2.6557 & 5.43 & 2.62671966 & 5.343644844 \\
\hline
\end{tabular}

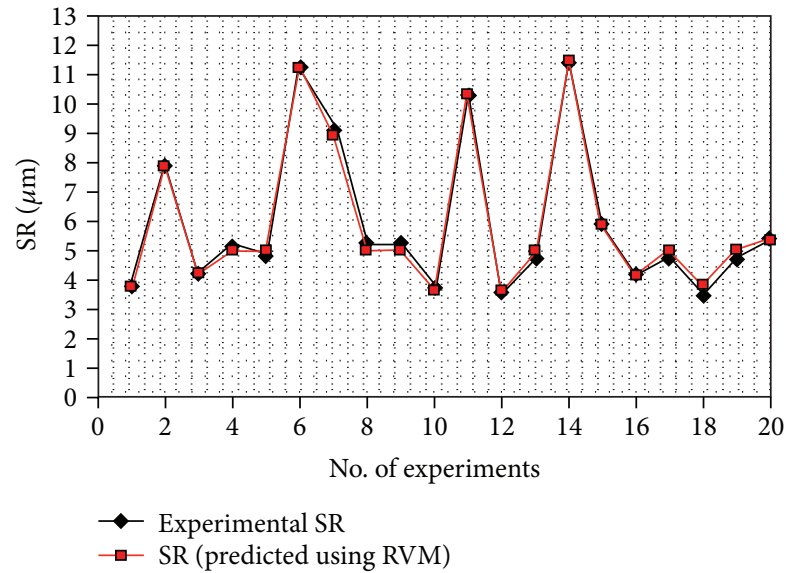

FIGURE 6: The comparison between predicted SR and experimental SR.

component in manufacturing industry. High value of pulse duration decreases the MRR. Roughing operation " $T_{\text {on }}$ " may be taken as $200 \mu$ s to $400 \mu \mathrm{s}$. The predicted values of MRR and SR from RVM model were found to be good and agreed with experimental results. The performance of the model is determined by using regression test error. The value of regression test error is found to be 0.010694 and 0.015266 for MRR and SR. This optimized result obtained by using cubic kernel RVM with the noise factor as 0.01 and the number of iterations as 200. This shows RVM as the best prediction method with low regression test error, which mainly leads to reduce the time and cost of the EDM process for better product design in the industry. Regression test error may be further reduced by considering more numbers of inputs to the RVM model as training and also better selection of model parameters.

\section{Acknowledgments}

The authors would like to thank Department of Mechanical Engineering, National Institute of Technology, Rourkela, India, for providing the facility to conduct the experiment. Also, they are thankful to anonymous reviewers and their suggestions for helping them to improve the quality of the paper.

\section{References}

[1] P. K. Mishra, Nonconventional Machining, The Institution of Engineers Textbook Series, Narosa Publishing House, 2011.

[2] D. C. Montgomery, Design and Analysis of Experiments, John Wiley \& Sons, New York, NY, USA, 6th edition, 2005.

[3] M. M. Rahman, M. A. R. Khan, K. Kadirgama, M. M. Noor, and R. A. Bakar, "Modeling of material removal on machining of Ti-6Al-4V through EDM using copper tungsten electrode and positive polarity," World Academy of Science, Engineering and Technology, vol. 71, pp. 576-581, 2010.

[4] M. A. R. Khan, M. M. Rahman, K. Kadirgama, M. A. Maleque, and M. Ishak, "Prediction of surface roughness of TI-6AL-4V 
in electrical discharge machining: a regression model," Journal of Mechanical Engineering and Sciences, vol. 1, pp. 16-24, 2011.

[5] M. K. Pradhan and C. K. Biswas, "Modelling and analysis of process parameters on surface roughness in EDM of AISI D2 tool steel by RSM approach," World Academy of Science, Engineering and Technology, vol. 57, pp. 814-819, 2009.

[6] S. S. Baraskar, S. S. Banwait, and S. C. Laroiya, "Mathematical modeling of electrical discharge machining process through response surface," International Journal of Scientific and Engineering Research, vol. 2, no. 11, pp. 1-10, 2011.

[7] S. Prabhu and B. K. Vinayagam, "Modeling the machining parameters of AISI D2 tool steel material with multi wall carbon nano tube in electrical discharge machining process using response surface methodology," International Journal of Physical Sciences, vol. 7, no. 2, pp. 297-305, 2012.

[8] X. P. Li and S. H. Lee, "Study of the effect of machining parameters on the machining characteristics in electrical discharge machining of tungsten carbide," Journal of Materials Processing Technology, vol. 115, no. 3, pp. 344-358, 2001.

[9] K. M. Tsai and P. J. Wang, "Predictions on surface finish in electrical discharge machining based upon neural network models," International Journal of Machine Tools and Manufacture, vol. 41, no. 10, pp. 1385-1403, 2001.

[10] M. E. Tipping, "Sparse Bayesian learning and the relevance vector machine," Journal of Machine Learning Research, vol. 1, no. 3, pp. 211-244, 2001.

[11] M. N. Wernick, A. S. Lukic, D. G. Tzikas et al., "Bayesian Kernel methods for analysis of functional neuroimages," IEEE Transactions on Medical Imaging, vol. 26, no. 12, pp. 1613-1624, 2007.

[12] J. Quiñonero-Candela and L. K. Hansen, "Time series prediction based on the relevance vector machine with adaptive kernels," in Proceedings of the IEEE International Conference on Acustics, Speech, and Signal Processing, pp. I/985-I/988, May 2002.

[13] W. Caesarendra, A. Widodo, P. H. Thom, and B. S. Yang, "Machine degradation prognostic based on RVM and ARMA/ GARCH model for bearing fault simulated data," in Proceedings of the IEEE Prognostics and System Health Management Conference (PHM '10), IEEE, China, January 2010.

[14] G. Camps-Valls, M. Martínez-Ramón, J. L. Rojo-Álvarez, and J. Muñoz-Marí, "Nonlinear system identification with composite relevance vector machines," IEEE Signal Processing Letters, vol. 14, no. 4, pp. 279-282, 2007.

[15] R. L. Mason, R. F. Gunst, and J. L. Hess, Statistical Designed Analysis of Experiments with Applications to Engineering and Science, A John Wiley \& Sons Publication, John Wiley \& Sons, New York, NY, USA, 2nd edition, 2003.

[16] W. Caesarendra, A. Widodo, and B. S. Yang, "Application of relevance vector machine and logistic regression for machine degradation assessment," Mechanical Systems and Signal Processing, vol. 24, no. 4, pp. 1161-1171, 2010.

[17] M. Saarela, T. Elomaa, and K. Ruohonen, "An analysis of relevance vector machine regression," Studies in Computational Intelligence, vol. 262, pp. 227-246, 2010.

[18] K. C. Nayak, R. K. Tripathy, and S. R. Panda, "Relevance vector machine based prediction of MRR and SR for electrochemical machining process," International Journal of Mechanical Engineering and Technology, vol. 3, no. 3, pp. 394-403, 2012.

[19] C. M. Bishop and M. E. Tipping, "Bayesian regression and classification," in Advances in Learning Theory: Methods, Models and Applications, vol. 190 of Nato Science Series III: Computer and Systems Sciences, pp. 267-285, 2003.
[20] Y. Zhang, Y. Liu, R. Ji, and B. Cai, "Study of the recast layer of a surface machined by sinking electrical discharge machining using water-in-oil emulsion as dielectric," Applied Surface Science, vol. 257, no. 14, pp. 5989-5997, 2011.

[21] K. C. Nayak and R. K. Tripathy, “Taguchi integrated least square support vector machine an alternative to artificial neural network analysis of electrochemical machining process," IOSR Journal of Mechanical and Civil Engineering, vol. 1, no. 3, pp. 0110, 2012. 

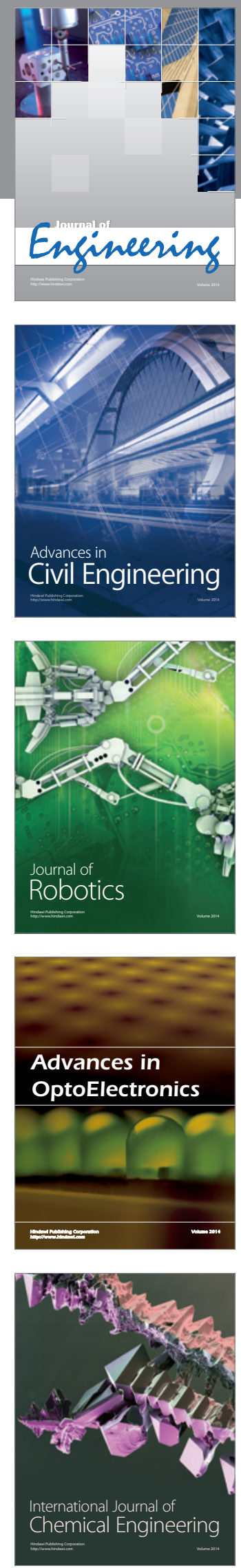

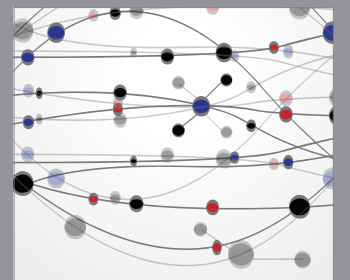

The Scientific World Journal
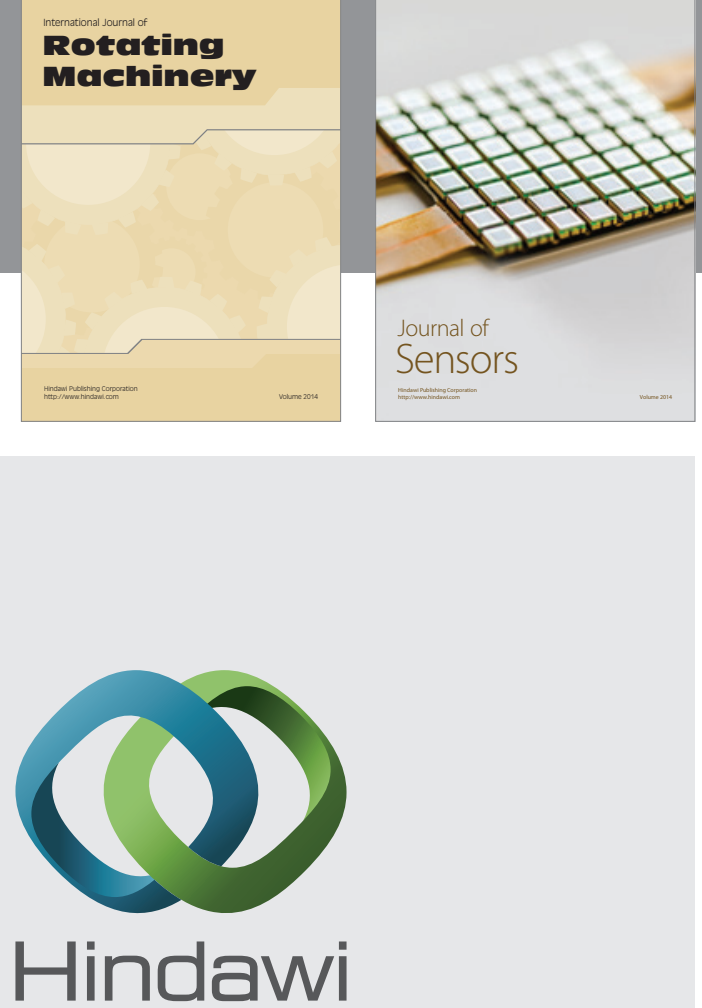

Submit your manuscripts at http://www.hindawi.com
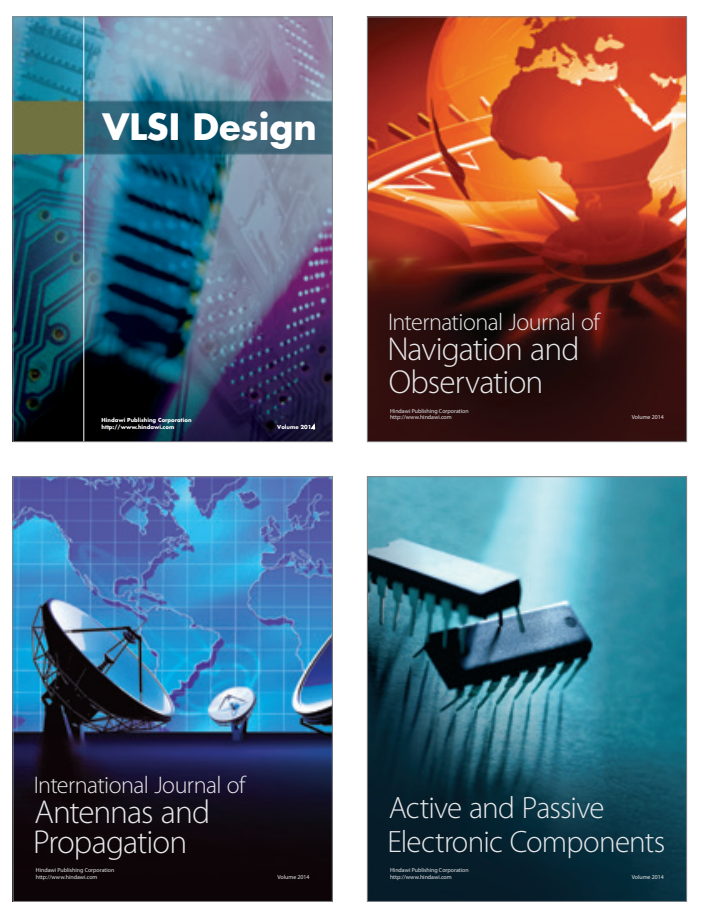
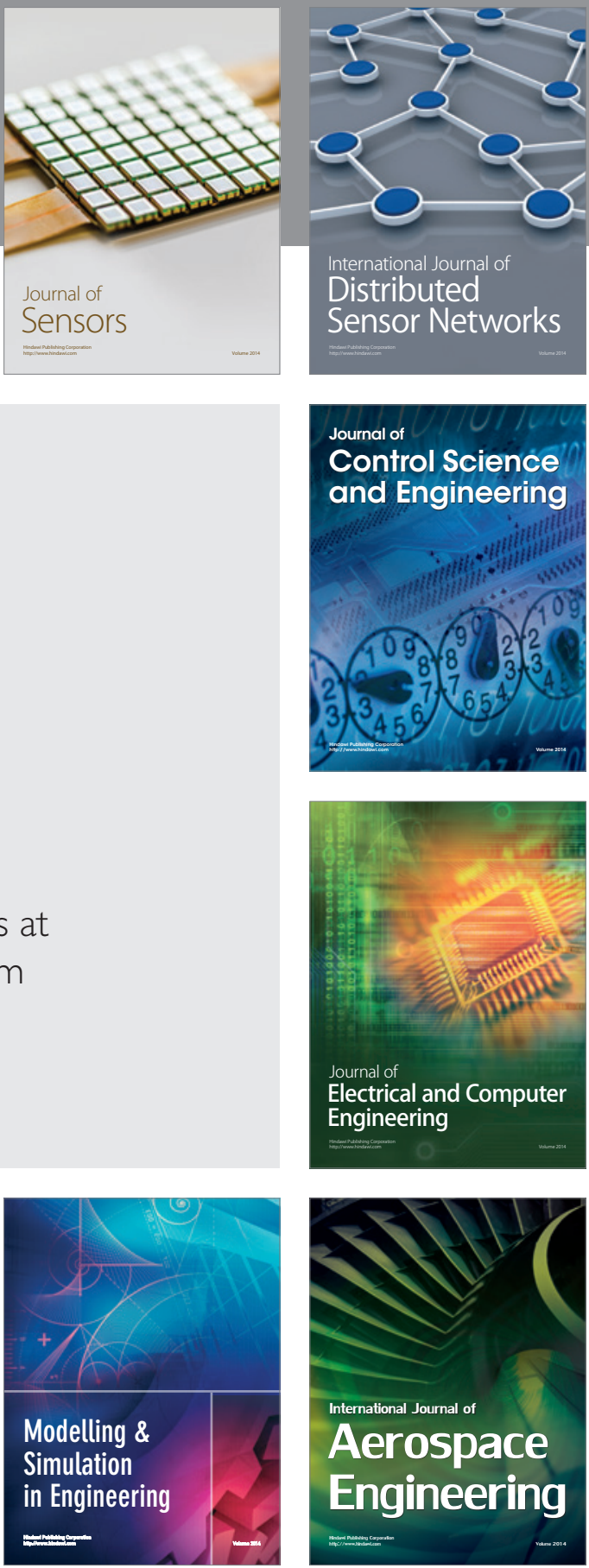

Journal of

Control Science

and Engineering
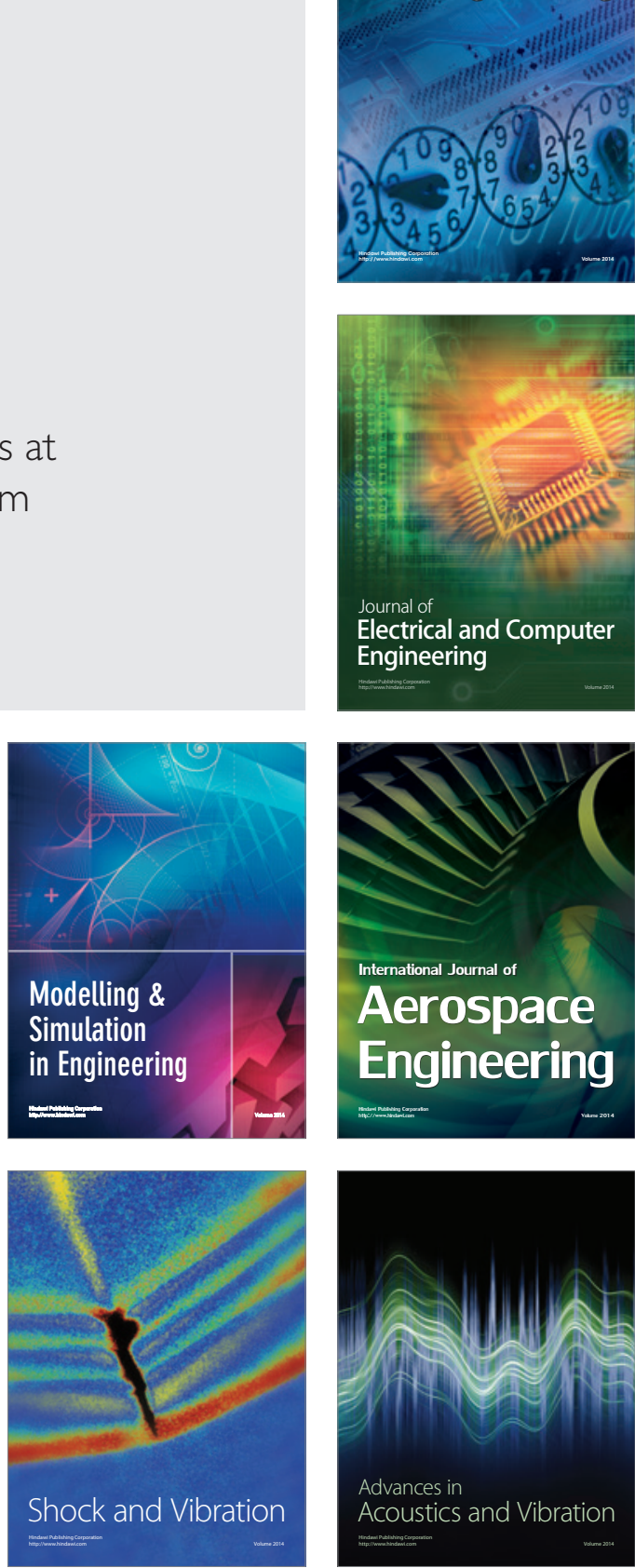